Joan-Rafael Ramos

\title{
La variación formal de los cuantificadores en catalán: estudio diacrónico (siglos XV-XX)
}

https://doi.org/10.1515/zrp-2020-0022

Abstract: This article studies the formal variation of the existential quantifiers $u$ / un ('one; a, an'), algú/algun ('someone; some'), ningú/ningun ('no one, anyone; any') and the distributive universal quantifier cada u/cada un ('everyone') in Catalan. The research is based on an extensive diachronic corpus of texts written between the 15th and 20th centuries. The author classifies the syntactic structures of these quantifiers and analyses their meaning and formal vacillations: apocopated form (without $-n$ ) vs. non-apocopated form (with $-n$ ). Although hesitations tend to disappear gradually during the period under analysis, the uses of these quantifiers change in Catalan dialects. In fact, distinct geographical areas adopt different distributions of form and function. These linguistic changes are explained, highlighting the mutual influences and interactions between phonosyntactic, phonological, analogical and pragmatic factors.

Keywords: linguistic variation, diachronic linguistics, linguistic change, morphology, quantifiers, Catalan

Palabras clave: variación lingüística, lingüística diacrónica, cambio lingüístico, morfología, cuantificadores, catalán

\section{Introducción}

Pertenecen a la categoría genérica de los cuantificadores la clase de palabras clasificadas tradicionalmente como numerales, cuantitativos e indefinidos. En este trabajo, centrado en el estudio de la lengua catalana, no abordaremos todo

\footnotetext{
Promovido por: Este estudio forma parte del proyecto de investigación Variación y cambio lingüístico en catalán. Una aproximación diacrónica según la lingüística de corpus, financiado por el Ministerio de Ciencia, Innovación y Universidades del Gobierno español (Ref. PGC2018099399-B-100).
}

Dirección de correspondencia: Prof. Dr. Joan-Rafael Ramos, Universitat de València, Departament de Filologia Catalana, Av. Blasco Ibáñez, 32, E-46010 València, E-Mail: joan.r.ramos@uv.es 
el conjunto de miembros de esta clase, sino que pretendemos estudiar aquellos que se han formado a partir del numeral latino unUM o de su combinación con otras bases léxicas. Se trata de las formas $u$, un, algú, algun, ningú, ningun, cada u, cada un.

La función que pueden desarrollar estos cuantificadores puede ser diversa (cf. Leonetti, 1999; Sánchez 1999; Rigau 1999; Brucart/Rigau 2002; Brucart 2002). Por un lado, pueden actuar como modificadores de un sustantivo. En este caso, ejercen una función de especificadores: se cuantifica sobre el núcleo de un sintagma nominal, el cual puede estar explícito (1), pero también puede tener un carácter implícito. En este segundo caso, la elipsis es recuperable discursivamente y se identifica con un referente concreto, específico (2). Tanto en un caso como en el otro existe la posibilidad de flexión de género y de número. ${ }^{1}$

(1) Ha entrat algun [home].

(2) a. Algun $\left[\emptyset_{\mathrm{i}}\right]$ dels [assistents $\left.\mathrm{s}_{\mathrm{i}}\right]$ va caure malalt.

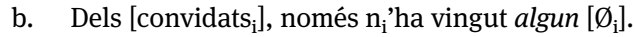

Por otro lado, pueden constituirse como elementos nucleares. No cuantifican sobre ningún otro elemento, sino que su valor queda integrado en su base léxica. En este caso, no se especifica sobre un constituyente concreto del discurso, sino que por sí solo aporta un valor inespecífico (3). Se trata, pues, de un pronombre, que, desde el punto de vista de la flexión, es invariable.

(3) Algú no ha pagat.

Esta distinción funcional entre especificadores (cuantificadores nominales) y elementos nucleares (pronombres), que hemos ilustrado en (1-3) con los cuantificadores algú y algun, también se puede aplicar al resto de los casos que queremos estudiar:

1 En este artículo no entraremos en el debate de si la naturaleza categorial de los cuantificadores en (2) es de carácter adjetival o pronominal (cf., por ejemplo, Brucart 2002, 1442; RAE/ASALE 2009, 1090-1098; IEC 2016, 571-572). La tradición gramatical, reflejada todavía en muchos diccionarios, suele considerar los usos de (2) con núcleo nominal implícito como pronombres, frente a los usos de (1) como adjetivos. Otras veces se habla de adjetivos determinativos en los dos casos, asumiendo que ejercen la misma función y que la diferencia entre ambos está en que en (1) el núcleo nominal está explícito y en (2) no lo está. Recientemente, ya son muchos los tratados gramaticales que, tanto en (1) como en (2), prefieren no usar el término adjetivo, y hablan de cuantificadores nominales, incluidos a menudo dentro de la categoría más general de los determinantes, a causa de su función como especificadores discursivos y no como complementos del nombre. En este trabajo, seguiremos este último criterio. 
(4) a. Ha entrat un home.

b. Un dels assistents va caure malalt.

c. Dels convidats, només n'ha vingut un.

d. Un no ha pagat.

(5) a. No ha entrat ningun/cap home. ${ }^{2}$

b. Ningun/cap dels assistents va caure malalt.

c. Dels convidats, no n'ha vingut ningun/cap.

d. No ha pagat ningú.

(6) a. Cada un dels assistents va caure malalt. ${ }^{3}$

b. Cada $u$ haurà de pagar les seues despeses.

Si nos centramos en la forma de masculino singular, que será el tema del presente estudio, los ejemplos de más arriba, propios de la lengua estándar, indican que los usos como especificador con núcleo explícito presentan siempre una solución terminada en $-n(1,4 a, 5 a)$; sin embargo, los usos como pronombre $(3,5 d, 6 b)$ muestran una solución formal apocopada (algú, ningú, cada u), excepto en el caso de un (4d), que es no apocopada. Asimismo podemos ver que los usos como especificador con núcleo implícito (2a-b, 5b-c, 6a) también contienen una forma no apocopada (algún, ningun, cada un), que les permite distinguir-se de los usos como pronombre, salvo en el caso de un (4b-c), que es formalmente sincrética.

Esta distribución formal, fijada por la normativa gramatical (Fabra 2010 [1923]; IEC 2016, 619-665), no acaba de ser del todo estable en la lengua hablada actual. De hecho, esta inestabilidad, que afecta todos los cuantificadores estudiados, es más significativa en áreas geográficas del catalán como la valenciana, donde es posible encontrar sincretismo formal entre los usos como pronombre (7a) y los usos como especificador con núcleo nominal implícito (7b-c).

(7) a. Algú no ha pagat.

b. Algú $\left[\emptyset_{\mathrm{i}}\right]$ dels assistents $\mathrm{s}_{\mathrm{i}}$ va caure malalt.

b. Dels convidats ${ }_{\mathrm{i}}$, només $\mathrm{n}_{\mathrm{i}}{ }^{\prime}$ ha vingut algú $\left[\emptyset_{\mathrm{i}}\right]$.

A la vista de estas alternancias, y con el fin de comprender mejor el comportamiento actual de la lengua, nuestro objetivo es estudiar la evolución de estos cuantificadores desde la Edad Media hasta el siglo XX. Este estudio nos permitirá hacer un seguimiento diacrónico de las diversas variantes formales y apuntar los factores que han podido interactuar en su elección.

2 Actualmente, el uso de la forma ningun se considera dialectal. La forma estándar es cap. Véase $\S 3.3$.

3 Cabe advertir que no es posible en la lengua moderna utilizar cada un como especificador con núcleo explícito: ^Ho fan cada un any. 


\section{Aspectos metodológicos}

Los estudios diacrónicos existentes sobre el tema son poco detallados y parciales. Obras como el DCVB y el DECLC, y autores como Gulsoy $(1996,43)$ y Camus (en prensa) han señalado las alternancias formales en catalán medieval de los cuantificadores analizados, pero sin precisar los diferentes usos sintácticos ni hacer un seguimiento a lo largo de la historia del catalán. Por su parte, Ramos (2018) profundiza en dos de los cuantificadores estudiados, u/un y cada u/cada un, pero su investigación solo analiza textos del siglo XVII. Finalmente, Saragossà (2005, 136-143) y Sastre (2015) han insistido en la vigencia de la solución apocopada $u$ en el área dialectal valenciana, pero sin aportar datos sistemáticos de carácter histórico.

Ante la falta de sistematicidad y de profundidad de la bibliografía existente, nuestro propósito es estudiar la variación formal de las formas de masculino singular teniendo en cuenta su uso en las diferentes estructuras sintácticas apuntadas en el apartado anterior y su comportamiento a lo largo del periodo histórico seleccionado (del siglo XV al siglo XX).

Para llevar a cabo esta investigación, hemos tenido como fuente los siguientes corpus informatizados: Corpus Informatitzat del Català Antic (CICA), Corpus Informatitzat de la Gramàtica del Català Modern (CIGCMod), Corpus Informatitzat del Valencià (CIVAL) y Corpus Textual Informatitzat de la Llengua Catalana (CTILC). En el caso del CICA, hemos analizado todos los textos clasificados en este corpus como pertenecientes al siglo XV. ${ }^{4}$ En cuanto al CIGCMod, hemos centrado nuestra atención especialmente en el siglo XVII, pero sin descuidar la revisión de textos del siglo XVI y XVIII. ${ }^{5}$ Finalmente, el CIVAL nos ha permitido acceder a textos valencianos del siglo XIX, y el CTILC nos ha servido de fuente de textos del

4 El CICA incluye las principales obras de esta época, con un volumen de palabras en el siglo XV de más de tres millones. Entre los textos podemos encontrar: obras literarias como Tirant lo Blanch, Curial e Güelfa o Lo Somni de Bernat Metge; partes representativas de Lo Crestià de Eiximenis, dels Sermons de Vicent Ferrer; traducciones de obras como el Decameró, Quesits e perquens; abundante documentación administrativa y jurídica; libros de cocina; inventarios; obras religiosas, etc. Véase el apartado de créditos del portal de CICA.

5 Entre los textos analizados pertenecientes al CIGCMod, estudiamos de manera detallada: Dietari de la Generalitat de Catalunya (años: 1617-1619, 1644-1647); Diari de Joan Guàrdia; Dietari de Jeroni Pujades (años 1601-1625); Revisió de les Memòries de mossèn Curp, rector de Vilallonga dels monts; Dietari de Pere Pasqual; Diari de Jeroni Cros; Memòries de l'església de Sant Jaume de Perpinyà; Vertaderes traces de l'art de picapedrer; Ordinacions de la Universitat d'Eivissa; Dietari de Joaquim Aierdi; Dietari de Pere Joan Porcar; Processos d'Elda i Oriola (años: 1600-1707); Diplomatari de la Vall d'Andorra; Comèdia famosa de la famosa verge i màrtir Santa Bàrbara; y Manuscrits del Consell de les Paüls (años: 1593-1667). Cf. para las características de este corpus, Antolí (2018). 
resto del catalán de ese mismo siglo. ${ }^{6}$ En la selección de textos de todos los corpus, hemos tenido en cuenta la procedencia geográfica con el fin de determinar si esta variable ha tenido incidencia también en la opción formal.

\section{Descripción de los usos}

Antes de llevar a cabo la descripción de los usos de cada uno de los cuantificadores, es necesario comentar que, en la evolución del latín al catalán, hay una regla fonética general que afecta las palabras que terminan en $-\mathrm{NE}(\mathrm{M}) \mathrm{O}-\mathrm{NU}(\mathrm{M})$. En este tipo de palabras, después de la supresión previa de las vocales átonas (-E, -U), la -N que queda en posición final también se elimina (cf. Duarte/Alsina 1986, 82-84; Gulsoy 1996). De este modo, palabras como vinu, homine, Bonu, unu pasan a vi, home, bo, $u$ en posición externa o absoluta. Esta pérdida de la -N, según Gulsoy (1996), ya está consolidada en el siglo IX, aunque en textos de la zona septentrional del catalán (antiguas diócesis de Elna y de Girona), las alternancias persisten hasta finales del siglo XIV.

Esta evolución fonética se ve alterada cuando esta clase de palabras ocupa una posición interna y no externa, es decir, cuando aparecen precediendo a un núcleo nominal. En este caso, la nasal final se mantiene: un bon vi; algun home.

De acuerdo con esta regla fonética, el comportamiento inicial de los cuantificadores en los primeros siglos del periodo medieval debería ser el siguiente:

a) pérdida de la - $n$ en posición externa, es decir, en las construcciones en que funciona como pronombre (8a) y en las que funciona como especificador con núcleo nominal elidido ( $8 \mathrm{~b}-\mathrm{c})$; y b) mantenimiento en posición interna, o sea, cuando actúa como especificador con núcleo nominal explícito (9).

(8) a. No ha arribat ningú.

b. De carros, només n'hi ha $u$.

c. Ho farà algú dels tres convidats.

(9) Ha portat un pi.

6 Del CIVAL hemos seleccionado diversas obras del siglo XIX de los autores siguientes: Josep Bernat i Baldoví (El virgo de Visanteta, 1845; L'agüelo pollastre, 1859); Eduard Escalante (Milacre de la muda, 1855; Cheròni y Riteta, 1872; Barraca de lo Cabañal, 1872; Fuchint de les bombes, 1873; La Moma, 1874; Les tres Palomes, 1876; Les chiques de l'entresuelo, 1877; El chiquet del miracle, 1878); Constantí Llombart (Teodor Llorente. Apunts biogràfics, 1880; Poesies valencianes, 1872); y Joaquim Martí Gadea (Ensisam de totes herbes, 1891). Del CTILC, se repasan superficialmente todos los textos del año 1833 al 1900, pero se estudian en profundidad los textos siguientes de Antoni de Bofarull de este periodo: Memoria (1859); La orfaneta de Menargues ó Catalunya agonisant (1862); Costums que's perden y recorts que fugen (1880); y Discurs y memòria (1883). 
Veamos a continuación hasta qué punto la evolución de la lengua catalana mantiene esta regla fonética o va adaptándose a las soluciones que hemos descrito para la lengua estándar. En este sentido, nos centraremos de manera especial en su comportamiento cuando es un pronombre y cuando funciona como especificador con núcleo nominal elidido.

\subsection{El caso de $u /$ un (< UnUm)}

El cuantificador $u /$ un derivado de unum puede aparecer en tres estructuras básicas: a) como especificador de un núcleo explícito; b) como pronombre indefinido; y c) como especificador de un núcleo implícito (elidido). ${ }^{7}$

Como especificador de un núcleo explícito, desde la Edad Media hasta la lengua actual, presenta en general la forma no apocopada un (10a). De hecho, resulta muy rara la aparición con la solución apocopada $u$, que podemos ver en el ejemplo medieval que ilustramos en (10b): ${ }^{8}$

(10) a. E cridà un seu gran companyó qui havia nom Alí Baxà (Història de Jacob Xalabín, 54, 21).

b. E la dona, hoynt dir aquelles paraules al metge juheu, penssant-se ella que ell ho sabés, près a gitar hú gran suspir (Història de Jacob Xalabín, 61, 12).

Esta estabilidad contrasta con las alternancias que podemos encontrar con las estructuras en que el cuantificador aparece en una posición externa. Así, como pronombre indefinido, los textos del siglo XV muestran vacilaciones, tal y como ilustramos en (11).

(11) a. Se sdevench que hun jorn ella demanava ha hú en quina manera ella poria fer lo servey de Deú (Decameró, 1. ${ }^{a}$ parte, 277, 25).

b. Com li seria sdevengut un cars que cuydant dar a hun hauria dat a son companyó (Llibre de Cort de Justícia de València 1441-1442, f. 28v, 41).

De este modo, podemos observar que hay textos valencianos donde la variante apocopada $u$ tiende a predominar, como es el caso de obras como Tirant lo Blanch

7 En este artículo, en que nuestro objetivo es contrastar este cuantificador con los otros tipos de cuantificadores formados con unum, no estudiaremos su uso como sustantivo numeral: el número $u$, l'u. Tampoco estudiaremos los usos de $u$ /un correlativos: (l')u/un...l'altre...Se puede encontrar información de estos usos en el siglo XVII en Ramos (2018).

8 En todos los ejemplos históricos que citaremos de ahora en adelante respetamos escrupulosamente las convenciones gráficas de los textos originales. Asimismo, la manera de citar la fuente del ejemplo sigue también los criterios específicos del corpus que se utiliza como base. 
(100\% de los usos con $u$ ) o Sermons de Vicent Ferrer (100\% de los usos con $u$ ); pero también hay otras obras en que la forma no apocopada un supera la forma apocopada: Epistolari de la València medieval (83,3\% de un) o Curial e Güelfa (100\% de un). ${ }^{9}$ En textos no valencianos conviven también las dos variantes, aunque, exceptuando la traducción del Decameró, con un 100\% de casos de $u$, la tendencia es preferir la solución no apocopada un. Por ejemplo, el Dotzè del Crestià contiene el 100\% de los usos con un, el Epistolari de Ferran d'Antequera un $100 \%$, Quesits e perquens un $100 \%$, Flors de Virtut un $90 \%$ o el Llibre de les Solemnitats de Barcelona un 66,6\%.

En los usos como especificador con núcleo nominal elidido, los textos del siglo XV muestran también continuas vacilaciones, de manera que es difícil establecer tendencias según la procedencia geográfica del texto, ya que diferencias territoriales no implican necesariamente una elección diferente, tal y como mostramos en (12), con ejemplos de origen catalán, y en (13), con ejemplos valencianos.

(12) a. Que la dita ciutat se servís del dit drap e que aprés, com se·n hich trobassen de bells, que-n fos comprat hu (Llibre de les Solemnitats de Barcelona 3, 349, 2).

b. E dix que la dita Mingueta se clamave que li havien lavat un mantell e un parell de lansols. E ella dix-li que ella sabia qui-n tenia un (El cavalleril'alcavota, 127, 10).

(13) a. Per ço com Phelip és hui hú dels bells cavallers del món (Tirant lo Blanch, 430, 8).

b. E hun dels caçadors que la y véu entrar, descavalcà a la porta de la cova (Tirant lo Blanch, 1097, 37).

Tanto es así que podemos observar, por un lado, una preferencia por el uso de la forma apocopada $u$ en textos valencianos como Tirant lo Blanch $(93,2 \%)$ o Sermons de Vicent Ferrer (80\%), pero también en textos administrativos catalanes como el Llibre de Solemnitats de Barcelona $(74,4 \%)$, Dietari o Llibre de Jornades (95,2\%) o en la traducción del Decameró (91,1\%); y por otro lado, un uso preferente de la variante no apocopada un en textos valencianos como Recull d'exemples $e$ miracles (100\%) y Curial e Güelfa (100\%), o en textos catalanes como Dietari de la Generalitat de Catalunya (95,7\%), Flors de virtut (100\%) o en el texto balear Llibre de Mostassaf (100\%).

Estas vacilaciones formales siguen vigentes hasta finales del siglo XVIII. Las alternancias tanto en los usos pronominales (14) como en los usos como especifi-

\footnotetext{
9 Hemos incluido Curial e Güelfa dentro de los textos de procedencia valenciana de acuerdo con la opinión más generalizada entre los filólogos (cf. Ferrando 2018). No obstante, el tema de su asignación geográfica sigue siendo polémico. Por otro lado, el contraste entre los usos de este cuantificador en esta obra y el uso que se hace en obras de referencia valencianas como Tirant lo Blanch o Sermons de Vicent Ferrer, tampoco no zanja la polémica.
} 
cador con núcleo elidido (15) se pueden encontrar en textos de diversa procedencia geográfica. Pero los datos obtenidos en este periodo ya permiten establecer algunas tendencias más claras (cf. Ramos 2018, §3). Así, por ejemplo, en el siglo XVII, en los usos pronominales, los textos valencianos muestran un predominio claro de la variante apocopada $u$, con porcentajes superiores al $90 \%$ de los casos (14a); en cambio, los textos no valencianos, continúan vacilando con porcentajes cercanos (14b).

(14) a. Y Tàpia la donà a hu del mateix palàcio (Dietari de Pere Joan Porcar, 267, 21).

b. Les quatre estatues foren cremades, una de $h u$ del Camp de Tarragona que era anat a renegar Alger y avia renegat y citat, y propter contumaciam excomunicat no avia purgat sinó incistit per annum ab la excomunicatió. La altra de un de Conflent, mort en Fransa, luterà (Dietari de Jeroni Pujades, vol. 15, 203, 18-21).

En cuanto a los usos como especificador con núcleo elidido, en este periodo de la Edad Moderna, los textos valencianos muestran un uso mayoritario de las soluciones con $u$ (94\% de los casos) (15a); por el contrario, los textos no valencianos presentan un menor uso de la solución apocopada y mayores vacilaciones (15b-c).

(15) a. Perseguint als matadors, en derrocaren hu en una escopetada (Dietari de Joaquim Aierdi, 418, 5).

b. Y entre molts lamps que lensà n'i hagué $h u$ que ferí en lo Monastir de les Gerònimes (Dietari de la Generalitat de Catalunya 1644-1647, 162, 39).

c. Quan pa tinguéren, que sols ne remangué un per deu o dotse personas (Diari de Joan Guàrdia, 81, 26).

Así, textos de la zona del catalán central, como el Dietari de la Generalitat de Catalunya, el Dietari de Jeroni Pujades y el Diari de Joan Guàrdia, aunque mantienen todavía un elevado uso de la forma apocopada, en conjunto superan el $40 \%$ de los usos con un. Este incremento de la variante un es más significativo en textos de zonas como la balear o la rosellonesa, y especialmente la noroccidental, donde se alcanza el $75 \%$ de los casos. ${ }^{10}$

Cuando analizamos textos del siglo XIX, las vacilaciones históricas tienden a minimizarse y las tendencias observadas en épocas anteriores se consolidan. De hecho, los textos valencianos estudiados presentan, en los usos como pronom-

10 Entre los textos de origen no valenciano, cabe advertir que los de autoría algueresa (ciudad de Alguer, en la isla de Cerdeña), en un avanzado siglo XVIII, muestran una tendencia muy similar a la valenciana, es decir, predominio de la variante formal apocopada para la posición externa: «quatre abres de poma del Corpus, $h u$ de pruna primarenca» (El lèxic alguerès de l'agricultura i ramaderia, $669,23)$. De hecho, sigue siendo esta todavía la solución que presenta este aislado dialecto catalán en la lengua actual (Bosch 2002, 152-153). 
bre, como única solución, la variante $u(100 \%)$ (16a); esta forma apocopada también predomina en los usos como especificador con núcleo elidido (90,5\%) (16b), donde un tiene un uso testimonial (9,5\%) (16c). Por el contrario, en los textos de procedencia no valenciana, tanto el uso como pronombre (17a) como el uso como especificador (17b), lo ha asumido la variante un (100\%). ${ }^{11}$

(16) a. Quant ú no vol, dos no riñen (Joaquim Martí Gadea, Ensisam de totes herbes, 1891, 432).

b. Y al passar per ú dels pobles del trànsit (J. Marí Gadea, Ensisam de totes herbes, 1891, 496).

c. Un d'ells, anomenat Ximén Corella, en nom de tots li parla al Compte trist (Constantí Llombart, Poesies valencianes, 1872, 389).

(17) a. Fent ab lo front y'ls ulls lo que fa un quant s'avergonyeix (Antoni de Bofarull, $L a$ orfaneta de Menargues ó Catalunya agonisant, 1862, 34, 30).

b. Es un dels majors consols que tinch en ma trista soledat (Antoni de Bofarull, $L a$ orfaneta de Menargues ó Catalunya agonisant, 1862, 145, 19).

\subsection{El caso de algú/algun (< ALIQUNUM < ALIQUIS UNUM)}

Las formas algú y algun presentan usos sintácticos similares a los de $u$ y un. ${ }^{12} \mathrm{El}$ análisis de textos del siglo XV nos permite comprobar que la forma no apocopada algun, ya desde la Edad Media, es estable cuando especifica un núcleo nominal explícito (18a), es decir, en posición interna. Por lo tanto, resulta raro encontrar en esta posición ejemplos como el de (18b) con la forma apocopada: ${ }^{13}$

11 Una ampliación del corpus de estudio tampoco parece que haga alterar esta tendencia. De hecho, un análisis de una de las construcciones con núcleo elidido, la formada por pronombre clítico en $+\mathrm{V}+\mathrm{u} / \mathrm{un}[\emptyset]$, en todo el corpus de textos no valencianos que integra el CTILC desde 1833 hasta 1901, nos indica que, de los más de 500 casos documentados, solo en 11 casos aparece la variante formal apocopada $u$.

12 Al lado de las formas algú y algun, en catalán medieval también podemos encontrar las variantes formales alcú y alcun. Por otro lado, todas estas formas, además de los usos sintácticos descritos en este trabajo, podían aparecer como complemento del nombre en entornos sintácticos negativos. En esta posición externa, siguiendo la regla fonética general, predominaba claramente la solución apocopada algú, aunque esporádicamente podía aparecer la alternancia con la forma no apocopada algun: «axí eren tots romasos freturosos de compaya que offici algú no podien ffer» (Decameró, 1. a parte, 12, 24); «ni de menes de soffre ni de foch algun» (Memorial del pecador remut, 158, 22). Estos usos, que también se documentan en la Edad Moderna, no serán objeto de estudio en este artículo.

13 También son raros los usos de la forma apocopada cuando la posición interna es fruto de la presencia de otro cuantificador nominal como altre: «si en lo dit raonament entrevenc nengú o si algú altre oý lo dit raonament» (El Cavaller i l'alcavota, 158, 8). 
(18) a. Si hi fan algun dampnatge, o hi ha algun empatxament (Llibre del Mostassaf de Mallorca 09, 256, 12).

b. E aprés spay de algú temps, lo comte En Griffa Pelós anà en França (Històries $e$ conquistes del realme d'Aragó e Principat de Catalunya, 202, 15).

La situación es diferente cuando el cuantificador estudiado ocupa una posición externa. En este caso, el análisis que hemos llevado a cabo de los textos del siglo XV nos permite observar que, como pronombre, la solución claramente predominante es la apocopada algú (19a), en general con independencia del origen geográfico del texto. No obstante, también se documentan ejemplos de alternancias con algun (19b), más significativas en textos de origen rosellonés o balear. Así, por ejemplo, frente a un texto valenciano como Tirant lo Blanch, que contiene un $100 \%$ de los usos con algú, otras obras catalanas como Lo Somni de Bernat Metge o el Dotzè del Crestià de Eiximenis muestran algunas alternancias con algun (respectivamente, un 11,3\% y un 8,5\% de casos de algun), y un texto administrativo balear como el Llibre del Mostassaf de Mallorca manifiesta un comportamiento apartado de la tendencia general, ya que el porcentaje de usos de algun alcanza el $71,4 \%$.

(19) a. Car impossible és que algú pogués ligar ne arrear fembres a lur guisa (Bernat Metge, Lo Somni, 198, 15).

b. Racional és: no·m pens que algun hi dubta quant veu que tracta coses divinals, sap les humanals, aprèn moltes arts e nobles disciplines, e, per rahó, tots los animals sobrepuja (Bernat Metge, Lo Somni, 76, 5).

En este mismo periodo del siglo XV, los usos como especificador de un núcleo nominal implícito también reflejan alternancias entre las formas algú y algun. Pero, en general, la solución apocopada algú suele predominar en la mayoría de los textos analizados (20a). A pesar de eso, cabe notar que el uso de algun (20b) es más importante que en las estructuras con pronombre, en especial en textos no valencianos como Lo Somni de Bernat Metge (100\% de los casos), Epistolari de Ferran d'Antequera (72,7\%), Quesits e perquens (75\%) o Llibre del Mostassaf de Mallorca $(100 \%){ }^{14}$

14 Entre las obras con uso generoso de la forma algun, también podemos destacar Curial e Güelfa, que vuelve a optar por una solución formal menos común entre los textos valencianos. De hecho, algun representa el $41,2 \%$ de sus usos pronominales y un $75 \%$ de sus usos como especificador con núcleo elidido. Estos porcentajes contrastan, por ejemplo, con el Tirant lo Blanch, que no tiene ningún uso de algun en estas dos construcciones estudiadas. 
(20) a. que laugerament se poria seguir ésser nafrat o alesiat de algú de vostres membres (Tirant lo Blanch, 300, 38).

b. denuncia e mana a tots e sengles cirurgians e barbers de la dita Universitat e Regne, que [....]. E si algun serà, qui aquells pagar no puxa, stiga tres jorns en la presó (Llibre del Mostassaf de Mallorca 11, 299, 34).

En el periodo del siglo XVI al XVIII, el corpus analizado nos indica que continúan en la lengua escrita las alternancias, pero con una tendencia en los usos pronominales a la consolidación de la forma apocopada algú. De hecho, la muestra de textos del siglo XVII que estudiamos con más detalle pone de manifiesto un uso exclusivo de la forma algú en los pronombres, tanto en textos valencianos como del resto del territorio lingüístico (21). En cambio, en las estructuras con núcleo elidido, a pesar de que continúan siendo mayoritarios los usos con algú en los textos valencianos y en los no valencianos (22a), el porcentaje de alternancias con algun es más significativo, ya que esta segunda variante oscila entre un 5 y un $50 \%$ de los casos (22b).

(21) Abans si moria algú no·n solian traurer altro (Dietari de Jeroni Pujades, vol. 15, 257, 33).

(22) a. Farà algú dels dits procehiments o comminarà fer-los (Dietari de la Generalitat de Catalunya 1617-1619, 404, 19).

b. Com fins vuy no se hage effectuat ni posat en exequució en beneffici de dita província algun de dits decrets, serà forsós altra vegada donar memorial (Dietari de la Generalitat de Catalunya 1644-1647, 24, 76).

La documentación estudiada del siglo XIX muestra una consolidación de la tendencia de tota la lengua a expresar por medio de la forma apocopada algú el pronombre, con alguna alternancia esporádica con algun (el 6,4\%, y solo en textos no valencianos). En cambio, cuando funciona como especificador con núcleo elidido, los textos muestran una tendencia que apunta ya claramente a los usos del siglo XX: por un lado, los textos valencianos se muestran conservadores, ya que mantienen de manera exclusiva (100\% de los casos) la forma apocopada algú (23a); por otro, los textos no valencianos continúan manifestando alternancias, pero la forma no apocopada algun se convierte en la más habitual (65,5\% de los casos) (23b).

(23) a. De corders se·n escapa algú, pero d'ovelles no·n queda cap (Joaquim Martí Gadea, Ensisam de totes herbes, 1891, 433).

b. Peróls áulichs forasters havian la major part desaparegut, y si algun ne quedava, tenia baixa sa vista (Antoni de Bofarull, La orfaneta de Menargues ó Catalunya agonisant, 1862, 366, 14). 


\subsection{El caso de ningú/ningun (< NEC UNUM)}

En cuanto al cuantificador formado a partir de la forma latina NEC UNUM, las formas antiguas más frecuentes correspondientes al catalán actual ningú/ningun son las variantes negú y negun. ${ }^{15}$

En la lengua medieval, el corpus analizado del siglo XV nos permite comprobar que estos cuantificadores existenciales pueden aparecer en posiciones sintácticas similares a las indicadas más arriba para su antónimo algú/algun. Así, cuando se usan en una posición interna, en general la solución formal que adopta es la variante no apocopada negun (24a), que se conservará hasta la actualidad, de tal manera que son raros, incluso en textos antiguos, los casos con la forma apocopada negú (24b). ${ }^{16}$

(24) a. Mas la persona que no·s té per digna de degun offici, tal serà exaltada (Vicent Ferrer, Sermons, vol. VI, 102, 19).

b. No volent-ho fer, lo prenguí sens nengú vituperi per los braços (Cartes triades dels Borja 2, carta 30, 77).

Por otro lado, si nos fijamos en los usos en posición externa, ${ }^{17}$ podemos decir que, en la lengua del siglo XV, en lo referente al uso nuclear como pronombre, la solución formal que predomina claramente es la apocopada negú. No obstante, también son posibles casos esporádicos de alternancias con negun, tal y como ilustramos dentro del fragmento reproducido en (25). Así, a modo de ejemplo, podemos señalar que la variante apocopada ningú ocupa porcentajes superiores al 95\% en obras como Tirant lo Blanch, Curial e Güelfa, Sermons de Vicent Ferrer, Lo

15 Estas formas conviven con otras variantes medievales como degú/degun, dengú/dengun, nengú/nengun que van abandonándose progresivamente hacia las variantes más modernas que sustituyen la vocal $e$ por la vocal $i$ : di(n)gú/di(n)gun, ni(n)gú/ni(n)gun (cf. también DECLC s.v.no; Camus en prensa, §15.5.5).

16 También son esporádicos los ejemplos con la variante apocopada cuando la posición interna es debida a la presencia de otro cuantificador nominal como altre: «per mijà algú sies vist star ab negú altre» (Tirant lo Blanch, 994, 4).

17 Como pasaba con las formas algú y algun, las formas negú y negun pueden desarrollar también un papel más propio de los adjetivos. Nos referimos a su uso pospuesto al núcleo nominal, en función de complemento del nombre. Se trata de una posición externa que, de acuerdo con la regla fonética general, debería adoptar la variante apocopada negú. De hecho, la solución generalizada en los textos analizados es esta: «Encara no havia dexeble degú que.l seguís tota hora» (Vicent Ferrer, Sermons, vol. 6, 183, 30). Y resultan extraños los casos con la forma no apocopada negun: «jamés d'allí avant no sentí en sí scalfament negun de la carn» (Recull d'exemples i miracles ordenat per alfabet, 240,12). Esta distribución formal en esta posición sintáctica se mantiene también en los textos de los siglos XVI al XVIII, y en el caso de textos valencianos incluso en el siglo XIX. 
Somni de Bernat Metge, Dotzè del Crestià, Dietari o Llibre de Jornades, Flors de virtut, entre otras; sin embargo, en otros textos como el Llibre del Mostassaf de Mallorca o el rosellonés Viatge d'en Ramon de Perellós al purgatori de Sant Patrici, la variante no apocopada negun tiene una mayor representación, respectivamente, $58,8 \%$ y $38,4 \%$, lo que sugiere un mayor uso de la forma no apocopada en zonas del Rosellón y de Baleares que en el resto del territorio de la lengua catalana.

(25) Que negú no gós nodrir oques, ànets ne pagos ne neguna altra volateria, mas que vull que cascú meng aytal carn com yo matex, ço és moltó, bou, vedell, cabró o crestat, cabrit o caça. Ne negun no gós menjar de neguna altra volateria (Eiximenis, Dotzè del Crestià, 1.- parte, vol. 1, 112, 22).

En cuanto a las estructuras sintácticas en que el cuantificador actúa como especificador con núcleo elíptico, cabe advertir que en los textos del siglo XV la variante que predomina claramente también es la apocopada negú (26), sin que ello impida algunas alternancias con negun (27). Por ejemplo, obras representativas como Tirant lo Blanch, Sermons de Vicent Ferrer, Curial e Güelfa, Lo Somni de Bernat Metge o Dietari o Llibre de Jornades contienen porcentajes de uso de negú del 100\%; otras como Dotzè del Crestià o L'Estudi General de Lleida superan el 70\%. A pesar de eso, en alguna obra la alternancia formal con negun es más significativa; este es el caso de obras como la valenciana Recull d'exemples i miracles ordenat per alfabet (57,1\% de casos de negun) o la obra rosellonesa de Regiment de sanitat a Jaume II (75\% de casos de negun).

(26) a. Ni qui negú d'ells no li acomanaria lo regiment de sa casa (Epistolari de la València Medieval II-3, carta 165, 46).

b. Lo dit mossèn Veguer, qui dix que no·n conegué negú (Regiment de sanitat a Jaume II, 173, 42).

(27) a. E per ço cor aquest mengar pot mol-lifficar lo ventre, deu ésser raebut ans que negun dels altres menjars (Regiment de sanitat a Jaume II, 163, 20).

b. De manera que no·n avem pogut perdre ningun (L'Estudi General de Lleida del 1430 al 1524 3, 186, 22).

En el periodo de la Edad Moderna, para los usos pronominales se consolida la variante ningú con independencia de la procedencia geográfica de los textos (28a). Por lo tanto, el uso de ningun en este tipo de construcciones es más bien residual (28b):

(28) a. Adverteix-se que ningú aportave dol sinó los tres verguers (Dietari de la Generalitat de Catalunya 1644-1647, 14, 68).

b. Que ningun los recullís, encara que fos pare y mare (Processos d'Eldai Oriola, 513,10). 
Por otra parte, como especificador con núcleo elidido también predomina claramente la solución apocopada ningú (29). Por ejemplo, los textos valencianos estudiados presentan un $83,3 \%$ de casos de ningú y los textos no valencianos un 90,9\%. Eso significa que la variante ningun, a diferencia de lo que ocurrió con algun, no progresa en estos contextos sintáctico-discursivos (30).

(29) a. Y de la campanya se tiraren tantes escopetades que de la part contrària de Domènech ne moriren onze y de la sua no·n morí ningú (Dietari de Jeroni Pujades, vol. 15, 207, 5).

b. Pues ningú de ells són estats recusats per a declarar (Dietari de la Generalitat de Catalunya 1644-1647, 108, 98).

(30) a. Sobra de valentia, no n'aguera ascapat ningun (Diari de Joan Guàrdia, 79, 13).

b. Pues aquell a ningun d'estos actes asistía, ni se n'adonava (Dietari de Pere Joan Porcar, 741, 19).

Este estancamiento de la variante ningun se tiene que enmarcar en el contexto de la aparición de una nueva forma más expresiva cap (CAPU < CAPUT) en la función de especificador nominal, documentada en los textos escritos a partir del siglo XVI. Este nuevo cuantificador es capaz de ocupar el lugar de ningú o de ningun en contextos sintácticos con núcleo nominal implícito (31), y el de ningun en estructuras con núcleo nominal explícito (32): ${ }^{18}$

(31) Perquè los castellans, en saber voluntaris, en ningun lloch fèian un destacament gros $i$, si cap ne podían agafar (Memòries de Francesc Gelat 1687-1722, 91).

(32) a. Y exhortant aquella, dit senyor jutje, y manant als ministres tirassen dix: «Senyor, jo no sé què-n diga, que no sé res, no sé cap de bruxa, may he morta cap de criatura» (Condemnades per bruixes. Processos judicials al Vallès i al Moianès a principis del segle XVII, 78).

b. Després diuhen se n'anà dita armada a Alicant y allí diuhen ne tiraren gran canditat de bombas, que a penas dexaren cap casa sencera (Memòries de mossèn Jaume Avellà 1674-1711, 32).

Cuando llegamos al siglo XIX, la variante ningú se convierte en exclusiva con el valor pronominal. Eso quiere decir que la tendencia observada en los siglos anteriores de preferencia por la forma apocopada ningú (33) queda completamente consolidada en este periodo (100\% de los casos de nuestro corpus):

(33) Ningú es vast si té dinés (Eduard Escalante, Les chiques del entresuelo, 1877, 5).

18 De hecho, el cuantificador cap, tanto con núcleo nominal implícito como con núcleo nominal explícito, es la forma más extendida en la lengua actual y la adoptada en la lengua estándar. Por otro lado, el ejemplo ilustrado en (32a) sugiere el origen del cuantificador, procedente de la estructura individualizadora cap 'cabeza' $d e+\mathrm{N}$ contable. 
Respecto a los usos como especificador de núcleo elidido, la presencia del nuevo cuantificador cap, tal y como hemos comentado más arriba, comporta una reducción significativa de las formas ningú y sobre todo de ningun. De hecho, en nuestro corpus solo hemos documentado casos de ningú (34): ${ }^{19}$

(34) a. Ningú dels presents s'ha sentat mes voltas en aquests banchs que jo, y ningú, per conseguent, es mes zelós que jo de la honra del Concell (Antoni de Bofarull, $L a$ orfeneta de Menargues ó Catalunya agonisant, 1862, 349, 39).

b. Y es lo pichor que consuelo pera la meua aflicsió no n'espere ningú (Josep Bernat i Baldoví, L'agüelo Pollastre, 1859, 14).

\subsection{El caso de cada u/cada un (< CATA UNUM)}

Otro de los cuantificadores formado con el numeral unum es el denominado cuantificador universal distributivo, cada u y cada un, que presenta como solución más habitual en la lengua antigua las formas cascú y cascun. ${ }^{20}$

En la lengua medieval, la posición de este cuantificador universal, como en el resto de los casos estudiados, podía ser interna y externa. ${ }^{21}$ El uso interno tenía como variante completamente consolidada, siguiendo la regla fonética general, la forma no apocopada cascun (35a), y resultaban extraños los ejemplos con la variante apocopada cascú (35b):

(35) a. Daven licència a cinch cavallers que cascun dia staven de sol a sol ab lo rey (Tirant lo Blanch, 1163, 8).

b. $\quad$ E ffarets de cascú corter ·IIII· hó ·V · trosos hó parts (Llibre de totes maneres de potatges de menjar, 174, 4).

En cuanto a la posición externa, este cuantificador puede actuar con un valor genérico, propio de la forma pronominal, o con un valor de especificador, por la elipsis del núcleo nominal. Este segundo uso, debido a su carácter universal, está restringido a las estructuras partitivas.

19 Una aproximación al corpus CTILC, más allá de los objetivos trazados en este artículo, nos permite observar que, entre el periodo de 1833 y 1900, todavía persisten casos de ningun en alternancia con los de ningú; ahora bien, restringidos a la construcción con complementos nominales partitivos (cf. con 33a): «á fi de que ningun de ells puga demanar la nullitat de dita venda» (Francesc Viladot, Documents notarials, 1833).

20 También podemos encontrar en los textos antiguos las variantes quiscú y quiscun o quescú y quescun (< QUISQUEM UNUM), y se han desarrollado formas mixtas como cadascú y cadascun. Cf. DECLC s.v. cada; Camus en prensa, \$15.4.2).

21 Recordemos que la posición interna se ha convertido en obsoleta en la lengua actual. 
Nuestra investigación de textos del siglo XV nos indica que, como pronombre, la forma que predomina claramente, con independencia del origen geográfico del texto, es la apocopada cascú (36a). Así se puede ver, por ejemplo, en obras como Tirant lo Blanch, Curial e Güelfa, Vita Christi de Isabel de Villena, Sermons de Vicent Ferrer, Lo Somni de Bernat Metge, Decameró, Quesits e perquens o Viatge d'en Ramon de Perellós al purgatori de Sant Patrici, que contienen un 100\% de los usos con cascú. Otras obras, a pesar de manifestar algunas alternancias con la forma no apocopada cascun, mantienen un porcentaje muy alto de usos de la variante apocopada cascú: Dotzè del Crestià (94,4\%), Flors de virtut (94,5\%), Diari o Llibre de Jornades (85,7\%). Finalmente, hay algunos textos con soluciones discordantes de la opción formal más general, en las que la solución predominante es la no apocopada cascun. Este es el caso, por ejemplo, de la traducción de los Aforismes de Hipócrates (con un 90,5\% de casos de cascun), o de obras como el Epistolari de Ferran d'Antequera o el Regiment de sanitat a Jaume II (36b), aunque estas dos últimas con un escaso uso del sentido genérico.

(36) a. Pens cascú en sí mateix quants pensaments e quantes vàries cogitacions abraçaren aquella dolorosa nit los dos amants (Curial e Güelfa, 54, 35).

b. Totes aquestes coses diligentment esguardades, porà cascun leugerament veer en qual manera dega usar les fruytes d'estiu e d'autumpne a regiment de sanitat (Regiment de sanitat a Jaume II, 143, 11).

Con respecto a los usos como especificador con núcleo elidido, la variante que domina en la mayoría de los textos estudiados es también la apocopada cascú. Como muestra, podemos señalar que obras representativas de la época como las siguientes tienen un porcentaje de usos de cascú superior al 95\%: Tirant lo Blanch, Curial e Güelfa, Sermons de Vicent Ferrer, Vita Christi de Isabel de Villena, Lo Somni de Bernat Metge, Decameró, Flors de virtut o Testament de Pere de Viana. También tienen usos superiores al 80\% obras como el Manual de consells de Gandia, el Dotzè del Crestià y el Llibre de Solemnitats de Barcelona (37a). En cambio, las alternancias son más significativas o incluso hay un repunte de los casos de cascun en obras como Recull d'exemples i miracles ordenat per alfabet (50\%), Història de la Corona i Regne d'Aragó (50\%), Quesits e perquens (66,6\%), Viatge d'en Ramon de Perellós al purgatori de Sant Patrici (66,6\%), Epistolari de la València medieval (75\%), Epistolari de Ferran d'Antequera (78,9\%) o Llibre del Mostassaf de Mallorca (100\%) (37b).

(37) a. En cascú dels dits altàs foren dites misses (Llibre de les Solemnitats de Barcelona 2, 241, 37).

b. Ab les respostes fetes per nós a cascun dels capítols de aquells (Epistolari de Ferran I d'Antequera, carta 108, 9). 
En el periodo de la Edad Moderna, se consolida la tendencia de los usos pronominales a seleccionar la forma apocopada cada $u$, con independencia de la procedencia del texto. Así lo pone manifiesto el corpus analizado, que muestra un uso exclusivo de esta forma (38).

(38) No volen ser correchits, sinó viure cada hu com vol (Dietari de Joaquim Aierdi, 404, 38).

Por otro lado, en los usos con elipsis de núcleo nominal, nuestra investigación pone de manifiesto que hay diferentes tendencias según la procedencia geográfica del texto. En los textos valencianos, predomina de manera absoluta la variante apocopada cada $u$; pero en los textos no valencianos, continúan las alternancias (39). Tal y como remarca también Ramos (2018, 613-614) en su estudio sobre el siglo XVII, en textos de esta procedencia, a pesar de continuar predominando la forma cada $u$, la presencia de casos con la forma no apocopada cada un se mantiene gracias a unos usos que oscilan entre el 5 y el 16\%, alcanzando en algunos textos valores superiores al $50 \%{ }^{22}$

(39) a. Anant-los a acompanyar a cada hu d'ells lo porter de son estament (Dietari de la Generalitat de Catalunya 1644-1647, 140, 25).

b. Los que sien menester, y, també, dits síndichs, o cada un de ells en particular (Diplomatari d'Andorra, 300, 11).

El análisis de textos del siglo XIX nos permite comprobar que se producen nuevos cambios en el comportamiento de las variantes estudiadas. En lo referente a los contextos como pronombre, cada $u$ está plenamente estabilizada en los textos valencianos (100\% de los casos); pero la variante cada un vuelve a alternar con cada $u$ en los textos no valencianos, en una distribución de usos similar, 50\% y $50 \%(40)$.

(40) a. Cuant lo de cada hu no es lo de tots (Antoni de Bofarull, Memoria, 1859, 32, 17).

b. La ma de la Providencia, que tè'l secret de saber lo que toca a cada un en aquest mòn (Antoni de Bofarull, La orfaneta de Menargues ó Catalunya agonisant, 1862, 91, 16).

En los usos como especificadores de un núcleo nominal implícito, los textos valencianos adoptan de manera exclusiva la variante apocopada cada $u$ (41). Por el contrario, los textos no valencianos manifiestan un aumento considerable de los

22 Esta vacilación está plenamente presente también a lo largo del siglo XVIII: por ejemplo, el estudio de una muestra, de 1769 a 1791, de la obra Calaix de Sastre del Baró de Maldà nos indica que el $72,7 \%$ de los casos son con cada u y el $27,3 \%$ son con cada un. Sin embargo, en la obra Dietari del setge de Barcelona de Manuel Soler, los casos de cada un representan el 100\%. 
usos de la variante no apocopada cada un, que supera el 70\% de los casos (cf. 42a y 42b).

(41) A cada ú dels cocheros deu realets li ha de dar (Joaquim Martí Gadea, Ensisam de totes herbes, 1891, 295).

(42) a. La fabricació de sederias, per medi de talers, cada hu dels quals estava á cárrech de un home (Antoni de Bofarull, Costums que's perden y recorts que fugen, 1880, $25,17)$.

b. De mica en mica s'anàren descubrint tresors en cada un dels brins d'aquellas herbas ans despreciadas (Antoni de Bofarull, Costums que's perden y recorts que fugen, 1880, 14, 3).

\section{Interpretación de la evolución}

Una vez hecha la descripción diacrónica de las variantes formales de los cuantificadores estudiados es el momento de abordar qué factores han podido intervenir en su selección y su fijación. En este sentido, interpretamos que han interactuado factores pragmático-discursivos, analógicos y fonológicos, que han tenido una incidencia diferente según las zonas geográficas. De hecho, como hemos podido ver en los apartados anteriores, gran parte del catalán resuelve las alternancias siguiendo fundamentalmente un criterio de distinción funcional; en cambio, la zona valenciana lo tiende a hacer adoptando un criterio fonosintáctico (cf. §4.4).

\subsection{Factores pragmáticos y discursivos}

El hecho de estabilizar las alternancias formales, como ocurre en gran parte del catalán, atendiendo a un criterio funcional está relacionado con la intervención de factores de carácter pragmático y discursivo. Obsérvese que en la lengua antigua el hablante podía expresar tanto con una variante formal como con la otra (u/un, alcú/ alcun, negú/negun, cascú/cascun) las mismas funciones y los mismos valores: a) la función nuclear, como pronombre, con un valor no específico; b) la función de especificador de un núcleo nominal elidido, es decir, como cuantificador nominal, con un valor específico y discursivamente correferente. Por lo tanto, por un lado, se trataba de formas sinónimas, ya que podían alternar, y por otro, de formas polisémicas, porque podían tener más de un significado. Esta situación, especialmente cuando el correferente discursivo estaba alejado o en las construcciones partitivas con complemento nominal tácito, debía de originar, a veces, contextos ambiguos en cuanto a la interpretación del cuantificador con un valor no específico o con un 
valor específico. Este es el caso, por ejemplo, de (43), donde se hace difícil saber si algú contiene un valor genérico o remite a un referente anterior como ells.

(43) E sobtosament se escampà la nova fora la sglésia, e entre los hòmens, la qual pervenguda a les horelles del marit qui detràs ells era, sens escoltar consolació e confort d'algú per lonch espay plorà (Decameró, $1 .^{\mathrm{a}}$ parte, 348,1 ).

El interés por evitar contextos de ambigüedad, tal y como se ha insistido desde perspectivas teóricas como la lingüística cognitiva (Traugott/Dasher 2002; Traugott 2012), es una de las causas principales del cambio lingüístico. Y en el caso que nos ocupa, puede explicar la especialización formal de las variantes estudiadas. Nótese que el hablante escoge formas diferentes para designar funciones y valores diferentes. En este sentido, también cabe advertir que no se elige una forma léxica diferente, sino una variante formal con la única diferencia de incorporar una - $n$ final o no. Este fenómeno conecta con el principio de la iconocidad de las formas, que a su vez se relaciona con la teoría del marcaje: ${ }^{23}$ las formas con más relevancia pragmática tienden a destacarse formalmente.

En nuestro caso, se utiliza la marca icónica de la - $n$ final como estratégica discursiva para señalar la opción más compleja: falta de núcleo nominal, correferencia discursiva, variación morfológica (género y número) y designación de elementos humanos y no humanos. Y se mantiene sin marca la opción más simple, la que tiene un carácter nuclear, un valor semántico no específico inherente, invariabilidad morfológica y designación solo de elementos humanos. El aumento progresivo a lo largo de la historia de este marcaje consolida esta estrategia discursiva, que finalmente queda convencionalizada y asumida por la norma académica.

Este interés por distinguir formalmente estas dos funciones no es exclusivo del catalán. En el panorama romance, por ejemplo, tenemos casos como el del español (algún frente a alguien; ningún frente a nadie), el del portugués (algum frente a alguém; nenhum frente a ninguén); el del gallego (algún frente a alguén; ningún frente a ninguém; cada un frente a cadaquén) o el del rumano (vreunul frente a cineva; niciunul frente a nimeni). Y en el caso más específico del indefinido negativo, también podemos encontrar distinciones, por ejemplo, en occitano (cap/degun frente a degü), en francés (aucun frente personne) o en sardo (nudda frente a neune). ${ }^{24}$

23 Sobre el principio de iconocidad y su relación con la teoría del marcaje, son interesantes las reflexiones de Dressler (1987, 7-8), Pérez Saldanya (1998, 13-33), Mithun (2003), Bybee (2003) o Van Langendonck (2007).

24 Para la información sobre las lenguas romances, cf. RAE/ASALE (2009, 1377-1501); Cunha/ Cintra (2008, 370-381); DRAG; Grevisse/Goosse (1993, 927-954 y 1076-1118); Alibèrt (1976, 8395); Jones (1993, 30-52, 203-213); Serianni (1991, 286-308), Lamuela (2005). 


\subsection{Factores analógicos}

Otro de los fenómenos con el que nos encontramos es la homogeneización por medio de las variantes con $-n$ de las formas que actúan como especificadores con núcleo explícito y de las que lo hacen como especificadores con núcleo implícito. Recordemos que, en este último caso, partíamos de las variantes apocopadas, sin - $n$, pero ahora nos encontramos con las variantes no apocopadas, con $-n$, lo cual permite, por un lado, establecer, como hemos visto, una distinción con respecto a las formas pronominales, pero, por otro, confiere homogeneidad a todos los usos como especificador nominal. Eso quiere decir que, para consolidar esta distinción entre pronombre (núcleo) y especificador nominal, entró también en acción uno de los factores más importantes en morfología, el de la analogía (Dressler 1987). Así, las formas del especificador, al margen de si el núcleo nominal es explícito o no, armonizan su forma optando por converger en la variante formal más habitual y representativa, la que acompañaba un núcleo explícito (cf. Bybee 2007).

Esta homogeneidad formal, no es extraña tampoco en el marco de las lenguas romances. Por ejemplo, en portugués, los cognados um, algum y nenhum ocupan las dos posiciones del especificador. Esto mismo ocurre en gallego con los cognados un, algún y ningún. Con todo, este sincretismo es más habitual con el cognado de un (también en occitano, francés y sardo) y con el cognado del indefinido negativo ningun (también en occitano, en francés y en sardo).

\subsection{Factores fonológicos}

A pesar de que la mayoría de los cuantificadores estudiados hayan tendido a especializar las variantes formales según la función, también hemos podido ver que, en el caso de $u / u n$, no se ha consolidado tal distinción. De hecho, los hablantes se han inclinado por el sincretismo formal. La razón de este comportamiento peculiar, a nuestro entender, puede explicarse atendiendo a factores fonológicos (cf. Ramos 2018, 614-616).

Siguiendo la regla evolutiva general del catalán, tal y como hemos señalado anteriormente, en posición externa las palabras terminadas en - $n$ perdían esta consonante (cf. §3). Esta pérdida, en el caso del resto de los cuantificadores estudiados, comportaba la formación de palabras con dos sílabas (alcú; negú; cascú); sin embargo, en el caso de los derivados de unum, la solución era una palabra de una sola sílaba $(u)$. Si al hecho de ser un monosílabo añadimos que está formado solamente por una vocal sin el refuerzo de una consonante trabada, el resultado es la existencia de una palabra capaz de funcionar como núcleo, es decir, tiene carácter léxico y es tónica, pero con muy poca base fónica. Esta escasez de mate- 
rial fónico en palabras nucleares resulta rara en catalán. Por lo tanto, el hablante intenta buscar una solución morfológica más acorde con las secuencias monosilábicas mínimas. ${ }^{25}$ Esta solución se encuentra recurriendo tempranamente a la variante con la - $n$ final (un), tanto para expresar el valor genérico como el específico, de tal modo que esto permite trabar la vocal y fortalecer, desde un punto de vista fónico e icónico, el cuantificador. Ante este panorama, la posibilidad de distinción entre $u$ y un en base a razones pragmáticas queda abortada.

En esta línea de fijación de la forma no apocopada un, ya generalizada en el siglo XIX, no podemos perder de vista tampoco el papel de la analogía. De hecho, existe una tendencia a la homogeneización paradigmática con el resto de las formas de los cuantificadores usadas en la posición de especificador con núcleo elidido (algun, ningun, cada un).

Por otra parte, nótese también que, en las lenguas romances, el sincretismo formal en esta clase de cuantificadores, tanto si son monosílabos como bisílabos, es bastante extenso. De este modo, podemos ver que lenguas como el portugués (um), el gallego (un), el occitano (un), el italiano (uno) y el español (uno) utilizan la misma forma para el especificador con núcleo elidido y para el uso pronominal.

\subsection{El caso del valenciano}

Una evolución diferente del resto del catalán es la que hemos visto en la zona valenciana. En esta área del catalán, las alternancias antiguas no evolucionan hacia una especialización formal basada en criterios pragmático-discursivos, sino que, en el siglo XIX y principios del siglo XX, se acaba estabilizando la selección de las variantes sobre la base de los criterios fonosintácticos que regían el paso del latín al catalán. Esto significa que tiene un uso de la lengua mucho más conservador, de tal modo que, en posición externa, en todos los cuantificadores estudiados, tanto si los usos son como núcleo pronominal como si son como especificador con núcleo nominal elidido, se adopta la variante apocopada sin nasal. Por el contrario, en posición interna, los usos se resuelven como en todo el catalán con $-n$.

25 Cabré Monné (1994; 1995), aludiendo al concepto de la minimidad de la palabra, ha destacado que no resulta extraño que las lenguas establezcan en sus palabras mínimos fonológicos que hay que cumplir o que se tiende a cumplir. En el caso del catalán, la secuencia fónica mínima de una palabra sería consonante + vocal y, de manera especial, una sola vocal, como por ejemplo nuestro caso $u$. Sin embargo, superarían estos mínimos las palabras monosílabas con vocal larga o diptongo y las constituidas por vocal trabada por una consonante, como es el caso de un (información personal de Jesús Jiménez). Cf. también Ramos $(2018,615)$. 
Este comportamiento del territorio valenciano enlaza con el de otras lenguas romances que se rigen por el criterio fonosintáctico, como el italiano, el sardo, el rumano y, en gran medida, también el español. Ahora bien, cabe advertir que, a lo largo del siglo XX, la distribución descrita para la posición externa no está exenta de vacilaciones. Por un lado, a las alternancias históricas residuales entre la forma apocopada y la no apocopada, vivas todavía en la lengua oral de la Comunidad Valenciana, se une la influencia de la lengua normativa y estándar. ${ }^{26}$ Este modelo favorece, especialmente en los textos escritos, la distribución de las variantes siguiendo el criterio funcional consolidado en el resto del catalán.

Por otro lado, la inestabilidad fonética observada históricamente para el caso del cuantificador $u$ también tiene incidencia en la zona valenciana. Pero, en este caso, la solución no se busca necesariamente en la variante no apocopada un, sino que se recorre a una opción de fuera del catalán que también tenga consistencia fónica. Nos referimos a la forma bisílaba uno del español. La presión de esta lengua sobre el catalán valenciano ha favorecido progresivamente el uso de la forma uno, en lugar de $u$, en buena parte del actual valenciano central y, especialmente, en la zona meridional de la Comunidad Valenciana, donde ya se puede encontrar alguna muestra incluso en textos de la Edad Moderna (44) (Ramos 2018, 604-605):

(44) En la casa dita de Boix que era la única heretat de dit territorio (al present sols queden fonamens y alguna paret) morí antigament uno que es portà a soterrar a esta església (Memòries d'un capellà del segle XVIII, 43, 7).

La opción por el bisílabo uno conecta con la observación que ha hecho Thomason (2003) en relación con el cambio fonético. Este autor indica que, en comunidades en que la lengua propia convive con otra lengua sobrevenida y socialmente prestigiada, a menudo se recorre a resolver problemas de inestabilidad fonética buscando la solución en la otra lengua. Pero cabe añadir que el fenómeno de la interferencia no termina aquí. Una vez asimilada, está dando pie a una incipiente extensión analógica a la morfología del resto de los cuantificadores estudiados, favoreciendo soluciones como las siguientes: algú > alguno, ningú > ninguno, cada $u>$ cada uno.

26 En este artículo, por razones de espacio, no entraremos en detalles sobre el comportamiento subdialectal de los cuantificadores en la Comunidad Valenciana. 


\section{Conclusiones}

Al principio de nuestro trabajo, hemos advertido que los cuantificadores compuestos con el derivado latino de unum, en la lengua hablada actual, no siempre tienen una solución morfológica homogénea cuando aparecen en una posición externa, a pesar de que hay una normativa gramatical establecida. Así, se puede observar casos de alternancia entre la variante formal no apocopada, con - $n$, y la variante formal apocopada, sin $-n$. Y esto no solo depende del tipo de construcción en que aparecen, sino también del origen geográfico de los hablantes. Ante estas alternancias hemos considerado que una investigación diacrónica de estos cuantificadores podría ayudarnos a entender mejor esta variación actual.

A través del estudio diacrónico hemos podido constatar que la lengua medieval presenta numerosas vacilaciones en cuanto a la selección de las variantes, tal y como ya habían apuntado algunos estudiosos. Con todo, el análisis pormenorizado de las diferentes estructuras sintácticas en que pueden aparecer estos cuantificadores nos aporta un dato inédito. Nos referimos al hecho de que, a pesar de las alternancias, las formas apocopadas, $\sin -n$, suelen predominar en las dos funciones propias de la posición externa: la nuclear (pronombre) y la especificadora con núcleo elidido (cuantificador nominal).

También es una aportación novedosa la descripción del comportamiento de estos cuantificadores desde la Edad Media hasta el siglo XX. En este caso, hemos señalado que en la zona valenciana se va estabilizando la variante apocopada, gracias al mantenimiento del criterio fonosintáctico que rige la pérdida de la $-n$ final en posición externa. Por el contrario, en el resto del catalán este criterio va quedando obsoleto. De hecho, en esta extensa zona, hemos visto que, excepto en el caso especial de la forma un, cada variante formal tiende a representar una función distinta. Así deja de tener transcendencia si ocupa una posición externa o no, y lo importante es si tiene un carácter sintáctico nuclear, forma apocopada, o si actúa como un especificador, forma no apocopada. Esta diferente distribución geográfica, a finales del siglo XIX, ya está consolidada.

Hemos comparado también el comportamiento del catalán con el del resto de las lenguas romances. Esto nos ha permitido ver qué similitudes y qué diferencias presentan estas lenguas. En este sentido, hemos visto que en algunas ocasiones el criterio que predomina es el fonosintáctico y en otras, sin embargo, es el funcional.

Finalmente, sin desatender los factores fonológicos y fonosintácticos que inciden en la evolución de la forma de los cuantificadores, así como el papel de la analogía como homogeneizadora del cambio, podemos decir que nuestra investigación contribuye a profundizar en la importancia de la pragmática en la interpretación del cambio lingüístico. En nuestro caso, la marca icónica de la - $n$ se ha 
aprovechado, en gran parte del catalán, como estrategia discursiva para evitar una posible ambigüedad entre el valor genérico y la identificación con un antecedente discursivo.

\section{Bibliografía}

Alibèrt, Loïs, Gramatica occitana: segon los parlars lengadocians, Montpelhièr, Centre d'Estudis Occitans, ${ }^{2} 1976$.

Antolí, Jordi, El procés de constitució del Corpus Informatitzat de la Gramàtica del Català Modern (CIGCMod). Objectius, criteris i avaluació, Notandum 48 (2018), 3-19, DOI: <http://dx.doi. org/10.4025/notandum.48.1> [último acceso: 01.07.2019].

Bosch, Andreu, El català a l'Alguer, Barcelona, Publicacions de l'Abadia de Montserrat, 2002.

Brucart, Josep Maria, Els determinants, in: Solà, Joan/Lloret, Maria-Rosa/Mascaró, Joan/Pérez Saldanya, Manuel (edd.), Gramàtica del català contemporani, vol. 2, Barcelona, Empúries, 2002, 1435-1516.

Brucart, Josep Maria/Rigau, Gemma, Els quantificadors, in: Solà, Joan/Lloret, Maria-Rosa/Mascaró, Joan/Pérez Saldanya, Manuel (edd.), Gramàtica del català contemporani, vol. 2, Barcelona, Empúries, 2002, 1517-1589.

Bybee, Joan, Mechanisms of change in grammaticization: The role of frequency, in: Joseph, Brian D./Janda, Richard D. (edd.), The handbook of historical linguistics, Oxford, Blackwell, 2003, 602-623.

Bybee, Joan, Diachronic linguistics, in: Geeraerts, Dirk/Cuyckens, Hubert (edd.), The Oxford handbook of cognitive linguistics, Oxford, University Press, 2007, 945-987.

Cabré Monné, Teresa, Condicions prosòdiques i minimitat en el tipus reduplicatiu «puput», Caplletra 19 (1994), 187-194, DOI: 〈https://doi.org/10.7203/caplletra.19.7371〉 [último acceso: 01.07.2019].

Cabré Monné, Teresa, Minimality in the Catalan truncation process, Catalan Working Papers in Linguistics 4:1 (1995), 1-21, <https://www.raco.cat/index.php/CatalanWP/article/view/ 73978> [último acceso: 01.07.2019].

Camus, Bruno, La quantificació. Els quantificadors, in: Martines, Josep/Pérez Saldanya, Manuel (edd.), Gramàtica del català antic, en prensa.

CICA = Torruella, Joan/Pérez Saldanya, Manuel/Martines, Josep (edd.), Corpus Informatitzat del Català Antic, <http://cica.cat> [último acceso: 05.07.2019].

CIGCMod = Martines, Josep, et al. (edd.), Corpus Informatitzat de la Gramàtica del Catalán Modern, en preparación [último acceso: 26.06.2018].

CIVAL = Corpus Informatitzat del Valencià, <http://cival.avl.gva.es/cival/buscador.jsp > [último acceso: 10.07.2019].

CTILC = Rafel, Joaquim (ed.), Corpus Textual Informatitzat de la Llengua Catalana, <http://ctilc. iec.cat> [último acceso: 05.07.2019].

Cunha, Celso/Cintra, Lindley, Nova gramática do português contemporâneo, Rio de Janeiro, Lexikon, ${ }^{5} 2008$.

DCVB = Alcover, Antoni Maria/Moll, Francesc de Borja, Diccionari Català-Valencià-Balear, 10 vol., Palma de Mallorca, Moll, 1964-1969, <http://dcvb.iecat.net/> [último acceso: 10.07.2019]. 
$\mathrm{DECLC}=$ Coromines, Joan, Diccionari etimològic i complementari de la llengua catalana, 10 vol., Barcelona, Curial, 1980-2001.

DRAG = Real Academia Galega, Dicionario da Real Academia Galega, <https://academia.gal/ dicionario> [último acceso: 28.06.2019].

Dressler, Wolfgang (ed.), Leitmotifs in natural morphology, Amsterdam/Philadelphia, Benjamins, 1987.

Duarte, Carles/Alsina, Àlex, Gramàtica històrica catalana, Barcelona, 2 vol., Curial, 1986.

Fabra, Pompeu, Converses filològiques 306, 308, in: id., Obres completes, vol. 7, Barcelona, Proa/Edicions 63/Edicions 3i4/Editorial Moll, 2010, 404-406 [reproducción del texto publicado en 1923].

Ferrando Antoni, El model lingüístic de Curial e Güelfa, Revista Valenciana de Filologia 11 (2018), 39-83, DOI: <https://doi.org/10.28939/rvf.v2i2.39> [último acceso: 20.06.2019].

Grevisse, Maurice/Goosse, André, Le bon usage, Paris, Duculot, ${ }^{13} 1993$.

Gulsoy, Joseph, El tractament de la - $n$ < n' en català, Caplletra 20 (1996), 33-82, <https://ojs.uv. es/index.php/caplletra/article/view/7352/10402> [último acceso: 20.05.2019].

IEC (Institut d'Estudis Catalans), Gramàtica de la llengua catalana, Barcelona, IEC, 2016.

Jones, Michael Allan, Sardinian syntax, London/New York, Routlegde, 1993.

Lamuela, Xavier/Ani, Virgil, El romanès. Estudi comparatiu entre la gramàtica del català $i$ la del romanès, Barcelona, Generalitat de Catalunya, 2006.

Leonetti, Manuel, El artículo, in: Bosque, Ignacio/Demonte, Violeta (edd.), Gramática descriptiva de la lengua española, vol. 1, Madrid, Espasa, 1999, 787-890.

Mithun, Marianne, Functional perspectives on Syntactic Change, in: Joseph, Brian D./Janda, Richard D. (edd.), The handbook of historical linguistics, Oxford, Blackwell, 2003, 552-572.

Pérez Saldanya, Manuel, Del llatí al català. Morfosintaxi verbal catalana, València, Universitat de València, 1998.

RAE (Real Academia de la Lengua Española)/ASALE (Asociación de Academias de la Lengua Española), Nueva gramática de la lengua española. Morfología y sintaxis I, Madrid, Espasa Libros, 2009.

Ramos, Joan-Rafael, Aproximació a la morfosintaxi dels quantificadors en el català del segle XVII, eHumanista/IVITRA 14 (2018), 597-620, <https://www.ehumanista.ucsb.edu/ivitra/volume/14> [último acceso: 10.05.2019].

Rigau, Gemma, Determinación y cuantificación del sintagma nominal, in: Bosque, Ignacio/ Demonte, Violeta (edd.), Gramática descriptiva de la lengua española, vol. 1, Madrid, Espasa, 1999, 311-362.

Sánchez, Cristina, Los cuantificadores: clases de cuantificadores y estructuras cuantificativas, in: Bosque, Ignacio/Demonte, Violeta (edd.), Gramática descriptiva de la lengua española, vol. 1, Madrid, Espasa, 1999, 1025-1028.

Saragossà, Abelard, Gramàtica valenciana raonada i popular, València, Tabarca, 2005.

Sastre, Ana, Estudi de les formes «un» $i$ «u» en l'obra de Fabra, Aula de Lletres Valencianes 5 (2015), 143-248.

Serianni, Luca, Grammatica Italiana. Italiano comune e lingua letteraria, Torino, UTET, ${ }^{2} 1991$.

Thomason, Sarah G., Contact as a source of language change, in: Joseph, Brian D./Janda, Richard D. (edd.), The handbook of historical linguistics, Oxford, Blackwell, 2003, 687-712.

Traugott, Elisabeth C., Pragmatics and language change, in: Allan, Keith/Jaszczolt, Kasia M. (edd.), The Cambridge handbook of pragmatics, Cambridge, Cambridge University Press, 2012, 549-566. 
Traugott, Elisabeth C./Dasher, Richard, Regularity in semantic change, Cambridge, Cambridge University Press, 2002.

Van Langendonck, Willy, Iconicity, in: Geeraerts, Dirk/Cuyckens, Hubert (edd.), The Oxford handbook of cognitive linguistics, Oxford, University Press, 2007, 394-418. 